\title{
LRIG1 negatively regulates RET mutants and is downregulated in thyroid cancer
}

\author{
DAVID LINDQUIST $^{1}$, FERNANDO C. ALSINA ${ }^{2}$, CARL HERDENBERG $^{1}$, CATHARINA LARSSON $^{3}$, \\ JO HÖPPENER ${ }^{4}$, NA WANG $^{3}$, GUSTAVO PARATCHA ${ }^{2}$, MIKLÓS TARJÁN ${ }^{5}$, \\ TIBOR TOT $^{5}$, ROGER HENRIKSSON ${ }^{1}$ and HÅKAN HEDMAN ${ }^{1}$
}

\author{
${ }^{1}$ Oncology Research Laboratory, Department of Radiation Sciences, Umeå University, SE-90187 Umeå, Sweden; \\ ${ }^{2}$ Institute of Cell Biology and Neuroscience (IBCN)-CONICET, School of Medicine, University of Buenos Aires (UBA), \\ Buenos Aires 1121, Argentina; ${ }^{3}$ Department of Oncology-Pathology, Karolinska Institutet, Cancer Center Karolinska, \\ Karolinska University Hospital, 17176 Stockholm, Sweden; ${ }^{4}$ University Medical Center Utrecht, \\ Division of Biomedical Genetics and Laboratory of Translational Immunology, 3508 GA Utrecht, The Netherlands; \\ ${ }^{5}$ Department of Pathology and Clinical Cytology, Central Hospital Falun, 79182 Falun, Sweden
}

Received November 21, 2017; Accepted January 24, 2018

DOI: $10.3892 /$ ijo.2018.4273

\begin{abstract}
Papillary thyroid carcinoma (PTC) and medullary thyroid carcinoma (MTC) are characterized by genomic rearrangements and point mutations in the proto-oncogene RET. Leucine-rich repeats and immunoglobulin-like domains 1 (LRIG1) is a suppressor of various receptor tyrosine kinases, including RET. LRIG1 expression levels are associated with patient survival in many cancer types. In the present study, we investigated whether the oncogenic RET mutants RET2A (C634R) and RET2B (M918T) were regulated by LRIG1, and the possible effects of LRIG1 expression in thyroid cancer were investigated in three different clinical cohorts and in a RET2B-driven mouse model of MTC. LRIG1 was shown to physically interact with both RET2A and RET2B and to restrict their ligand-independent activation. LRIGI mRNA levels were downregulated in PTC and MTC compared to normal thyroid gland tissue. There was no apparent association between LRIG1 RNA or protein expression levels and patient survival in the studied cohorts. The transgenic $R E T 2 B$ mice developed pre-cancerous medullary thyroid lesions at a high frequency $(36 \%)$; however, no overt cancers were observed. There was no significant difference in the incidence of pre-cancerous lesions between Lrigl wild-type and Lrigl-deficient RET $2 B$ mice. In conclusion, the findings that LRIG1 is a negative regulator of RET2A and RET2B and is
\end{abstract}

Correspondence to: Dr David Lindquist, Oncology Research Laboratory, Department of Radiation Sciences, Umeå University, SE-901 87 Umeå, Sweden

E-mail: david.lindquist@onkologi.umu.se

Key words: thyroid cancer, LRIG1, RET, C634R, M918T, MEN2A, MEN2B also downregulated in PTC and MTC may suggest that LRIG1 functions as a thyroid tumor suppressor.

\section{Introduction}

Thyroid carcinoma is the most common endocrine malignancy. In 2008, 212,000 new cases were diagnosed worldwide (1). Thyroid carcinomas are classified into three major morphological well-differentiated types: papillary thyroid carcinoma (PTC), follicular thyroid carcinoma (FTC) and medullary thyroid carcinoma (MTC) with overall good prognosis (2). In contrast, poorly differentiated thyroid carcinoma (PDTC) and undifferentiated anaplastic thyroid carcinoma (ATC) carry a poor prognosis (2). PTC, FTC, PDTC and ATC are believed to originate from follicular cells, whereas MTC is believed to be derived from calcitonin-producing parafollicular cells (also called C-cells). Multiple endocrine neoplasia type 2A (MEN2A) and 2B (MEN2B) are caused by germ-line mutations in the proto-oncogene rearranged during transfection (RET) and are also associated with an increased incidence of MTC (3). The most common RET mutations in MEN2A and MEN2B are C634R and M918T, respectively (4) (hereafter the correspondingly mutated receptors are referred to as RET2A and RET2B, respectively). Twenty-five to $30 \%$ of MTC cases are associated with heritable RET mutations (5). In sporadic MTC, somatic RET mutations, typically the M918T mutation, are present in $\sim 50 \%$ of cases (3). In PTC, genomic rearrangements involving $R E T$ and different partner genes are common, especially in radiation-induced cases (reviewed in ref. 5). Thus, the incidence of RET rearrangements in PTC ranges from $87 \%$, in a series of early cases from the Chornobyl nuclear accident (6), to 20 and $7 \%$, in two recent series of sporadic cases $(3,7,8)$. In most cases, thyroid carcinomas are localized to the thyroid gland and the treatment used for these patients includes surgery, radiation therapy and radioiodine therapy. For patients with metastatic disease, however, the treatment options are limited. 
The human leucine-rich repeats and immunoglobulin-like domains (LRIG) gene family includes three genes: LRIGI, LRIG2 and LRIG3 (9-11). Increasing evidence indicates that $L R I G 1$ functions as a tumor suppressor (reviewed in refs. 12 and 13) and the high expression of the LRIG1 mRNA or protein are associated with an increased survival in breast cancer (14), ovarian cancer (15), uterine cervical cancer (16), cutaneous squamous cell carcinoma (17), nasopharyngeal and oropharyngeal cancer $(18,19)$, non-small cell lung cancer (20), and hepatocellular carcinoma (21). Molecular studies have shown that LRIG1 negatively regulates wildtype tyrosine kinase receptors of the epidermal growth factor receptor (EGFR) family (22-25), hepatocyte growth factor receptor (MET) (26), RET (27), platelet-derived growth factor receptor $\alpha$ (PDGFRA) (28), and neurotrophic tyrosine kinase receptor type 2 (NTRK2, also known as TRKB) (29). However, it is not known whether the MEN2- and thyroid cancer-associated mutant RET receptors, RET2A and RET2B, are also LRIG1 targets.

The aim of the present study was to investigate the possible role of LRIG1 in thyroid carcinoma. We investigated whether LRIG1 regulates RET2A and RET2B at the molecular level, analyzed the expression and possible prognostic value of LRIG1 in three human thyroid carcinoma cohorts, and investigated the role of Lrigl in a transgenic RET2B-driven mouse MTC model.

\section{Materials and methods}

Cell culture, plasmids and transfections. COS-7 cells (ATCC, Manassas, VA, USA) were grown in Dulbecco's modified Eagle's medium supplemented with $10 \%$ fetal bovine serum, as previously described (29). The cells were transiently transfected with polyethylenimine-PEI (Polysciences, Inc., Warrington, PA, USA). Plasmid cDNA encoding full-length, Flag-tagged LRIG1 has been described previously (30). The expression vector encoding RET2A (C634R) was previously described (31). The cDNA expression vector encoding RET2B (M918T) was kindly provided by Dr M. Santoro at University Federico II (Naples, Italy) (32).

Immunoprecipitations and western blotting. The cells were lysed at $4^{\circ} \mathrm{C}$ in TNE-buffer (50 mM Tris pH 7.5, $150 \mathrm{mM} \mathrm{NaCl}$ and $2 \mathrm{mM}$ EDTA) supplemented with $0.5 \%$ Triton $\mathrm{X}-100$, $1 \%$ octyl- $\beta$-glucoside, phosphatase inhibitors $(50 \mathrm{mM} \mathrm{NaF}$, $2 \mathrm{mM} \mathrm{Na}_{3} \mathrm{VO}_{4}$ ), and complete EDTA-free protease inhibitors (Roche). Cell lysates were clarified by centrifugation and analyzed by immunoprecipitation and western blotting using previously described methodologies (27). Ligand-independent RET phosphorylation was examined at $48 \mathrm{~h}$ after transfection in COS-7 cells expressing RET2A or RET2B constructs together with either an empty vector or Flag-LRIG1. The blots were scanned in a Storm 845 PhosphorImager and quantifications were conducted with ImageQuant software (both from GE Healthcare Life Sciences, Capital Federal, Argentina). The antibodies used were anti-phosphotyrosine (p-Tyr, clone PY99, catalogue no. SC7020) at a 1:10,000 dilution, anti-Ret (goat antibodies C20 and T20, catalogue no. SC-1290) (both from Santa Cruz Biotechnology, Santa Cruz, CA, USA) at a 1:1,000 dilution, and anti-Flag M2 mouse monoclonal antibody (catalogue no. F1804; Sigma-Aldrich, St. Louis, MO, USA) at a 1:1,500 dilution.

Patient cohorts. Three different patient cohorts were investigated. The first cohort (Cohort1-TCGA) comprised the thyroid carcinoma cases available at The Cancer Genome Atlas (TCGA), which included 502 PTC and 58 normal thyroid gland tissues (http://cancergenome.nih.gov/; December 15,2016). The RNAseq count data and patient survival data were downloaded from TCGA (http://cancergenome.nih.gov/; December 15, 2016). The samples were upper quartile normalized FPKM (UQ-FPKM) and the samples were log-transformed to follow a normal distribution.

The second cohort (Cohort2-MTC) has been described previously (33) and included 39 MTC patient samples and 12 normal tissue controls collected with informed consent and local ethical approval at the Karolinska University Hospital. The clinical data, survival and mutation status of the RET and $R A S$ genes were as reported in a previous study (33).

The third cohort (Cohort3-TMA) was collected with the aim of including all patients diagnosed with thyroid carcinoma in the county of Dalarna, Sweden, between the years 1980 and 2008. The study was approved by the Regional Ethics Review Board in Uppsala (dnr 2010/351), and the Swedish Cancer Registry was used to identify the patients. Only patients who provided written informed consent were included in the study. Paraformaldehyde-fixed and paraffin-embedded tissues were retrieved from hospital archives and used to assemble a tissue microarray (TMA). The diagnostic groups PTC, FTC, MTC, PDTC and ATC were represented, and FTCs with or without oncocytic features (so called Hurthle cell or oxyphilic) were separately studied. All patients gave written informed consent prior to their inclusion in the study. The clinical data were collected from patient files, including histology, TNM stage, time to relapse, and date and cause of death.

$R N A$ extraction and quantitative real-time $R T-P C R$. The RNA from Cohort2-MTC, which had been prepared previously (33), was treated with TURBO DNA-free kit (Life Technologies) according to the manufacturer's instructions. Human LRIGI and the reference gene RNI8S were analyzed in triplicate samples of $20 \mathrm{ng}$ total RNA through quantitative reverse transcription (RT) polymerase chain reaction (PCR), essentially as previously described (9). However, in contrast to what was previously described (9), qScript 1-Step qRT-PCR Kit (Quanta Biosciences, Gaithersburg, MD, USA) and a Bio-Rad CFX96 apparatus (Bio-Rad Laboratories AB) were used. The RNI8S-normalized LRIGl levels were divided by the corresponding level in the QPCR Human Reference Total RNA (Agilent Technologies, Santa Clara, CA, USA). Thus, the normalized LRIGl level in the reference RNA was set to the value of 1 .

Antibodies and evaluation of immunohistochemistry. Immunohistochemistry (IHC) was performed for Cohort3-TMA. IHC was performed on 4- $\mu$ m TMA sections using the LRIG1-151 antibody $(18,30)$ at a dilution of $1: 50$. The antibody against human LRIG1 has been previously described. The intensity and proportion of positive cells were evaluated by an experienced pathologist (M.T.), who had previously performed a 
similar evaluation using the same semi-quantitative evaluation scale in another material (18). Briefly, the intensity was evaluated as absent $(0)$, weak $\left(1^{+}\right)$, intermediate $\left(2^{+}\right)$, or strong $\left(3^{+}\right)$, and the fraction of positive cells were evaluated and scored as $0 \%, 1-25 \%, 26-50 \%, 51-75 \%$ or $76-100 \%$ positive cells.

Animal experiments. All mice were housed and maintained, and all experiments were performed in accordance with the European Communities Council Directive (86/609/EEC). The experimental protocols were approved by the Regional Ethics Committee of Umeå University in Umeå, Sweden (registration nos. A80-08 and A81-11). The animals were housed under controlled conditions with a 12-h day/night cycle and fed water and standard chow pellets (cat. no. 801730; Special Diets Services, NOVA-SCB Sweden, Sollentuna, Sweden) ad libitum. The Lrigl-deficient allele has been described previously (34) and was bred onto a pure C57BL/6J genetic background for five generations. Heterozygous Lrigl mice were crossed with a transgenic mouse strain that ectopically expresses the human RET2B oncogene under the human calcitonin promoter and develop MTC at a high frequency $(35,36)$. The RET2B mice were of a mixed genetic background (C57BL/6J:DBA2, 1:1). The mouse genotypes were determined using tail DNA, PCR, and standard molecular biology techniques. The PCR primers that were used for the Lrigl wild-type were: 5'-GCGAGCGTGTGTTGTGG AGAGGAT-3' and 5'-CGATTGTCTGGTGATCAGGAGAC $T G C-3$ ', those used for the Lrigl knockout were: 5'-ACCTCA GGGAGCGAGCGCTCTTATGGGTTAGGACG-3' and 5'-CCC GTGATATTGCTGAAGAGCTTGGCGGCGAATGG-3', and those used for the RET2B were: 5'-TGGAGACCCAAGACATC AAC-3' and 5'-GGAGAAGAGGACAGCGGCT-3'. The PCR products (405 bp for the Lrigl wild-type; 1,060 bp for the Lrigl knockout; and 220 bp for the RET2B) were separated and visualized using analytical agarose gel electrophoresis.

The plasma calcitonin levels of the mice were measured every third month according to the methodology as previously described (36). Plasma calcitonin is an established marker of MTC and increased levels of it are usually detectable long before general signs of the disease. At 2 years of age or at signs of the disease, the mice were sacrificed and their thyroid glands were dissected, fixed in $4 \%$ phosphate-buffered formaldehyde, embedded in paraffin, and stained with routine hematoxylin and eosin staining.

Statistical analyses. To analyze differential gene expression, the tumor samples were compared with normal samples using a Student's t-test in Cohort1-TCGA and a Mann-Whitney U test in Cohort2-MTC. Associations between ordinal variables were tested using the Wilcoxon rank sum test, $\chi^{2}$ test, one-way analysis of variance (ANOVA) not assuming equal variances, or Fisher's exact test, where appropriate. For survival analysis, the tumor samples were divided into two groups of high and low LRIG1, with the median as the cut-off, and the cumulative survival was then analyzed in the two groups and compared using the log-rank test. Survival was assessed in a Kaplan-Meier graph and a log-rank test was used for comparing different groups. Progression-free survival (PFS, the end-point date of relapse, censoring at the date of the last follow-up), disease-specific survival (DSS, the end-point date of death when thyroid cancer was specified as the cause of death, censoring at the date of the last follow-up), and overall survival (OS, the end-point date of death from any cause, censoring at the date of the last follow-up) were all addressed. The groups that were investigated included the expression of LRIG1, as well as other known prognostic factors, including clinical stage and age. All of the significance testing was performed at the 0.05 level and only two-sided P-values were presented.

\section{Results}

Interactions between LRIG1 and mutant RET2A and RET2B proteins. To investigate whether LRIG1 interacts with and regulates the oncogenic RET2A and RET2B receptors, COS-7 cells were co-transfected with LRIGl and the respective $R E T$ variant. After $48 \mathrm{~h}$, cell lysates were prepared and subjected to immunoprecipitation and western blot analysis. LRIG1 physically interacted with both RET2A and RET2B, as shown by the co-immunoprecipitation of LRIG1 and the RET2A and RET2B proteins (Fig. 1A and B). Furthermore, LRIG1 co-expression induced the downregulation of the phosphorylation levels of RET2A and RET2B without significantly affecting the corresponding total protein levels (Fig. 1C-F). Thus, LRIG1 physically interacted with and inhibited the activation of both RET2A and RET2B.

LRIGl gene and protein expression levels and comparison to clinical features. To investigate the importance of LRIG1 expression in thyroid cancer, we i) analyzed the PTC expression data available at TCGA (Cohort1-TCGA), ii) performed quantitative RT-PCR analysis of a series of MTC samples (Cohort2-MTC), and iii) analyzed a thyroid cancer TMA (Cohort3-TMA) through immunohistochemistry.

Cohort1-TCGA contained data from 502 PTC samples and 58 normal thyroid gland samples. In this material, the mean LRIGl expression level was lower in PTC than in the normal thyroid gland ( $\mathrm{P}=2.2 \times 10^{-16}$, Student's t-test) (Fig. 2A). There was a significant association between a higher PTC clinical stage and lower $L R I G 1$ expression $(\mathrm{P}=0.0034$, one-way ANOVA) (Fig. 2B). There was no significant association between the OS of patients and the LRIG1 expression level in the TCGA data set (high versus low LRIG1 expression level, median used as cut-off; $\mathrm{P}=0.435$, log-rank test).

In Cohort2-MTC, the relative LRIG1 mRNA levels were analyzed in 39 MTC and 12 normal thyroid glands through quantitative RT-PCR. Twenty-two of the 39 (56\%) MTC cases were RET mutated, whereas six (15\%) showed either HRAS or KRAS mutations (33). The intragroup variability of $L R I G 1$ expression was relatively high, as evidenced from the sample standard deviations from the means that were 179 and $70 \%$ for the normal and MTC samples, respectively. Nevertheless, the relative $L R I G 1$ expression level was significantly lower in MTC than in normal thyroid gland $(0.38, \mathrm{SD} \pm 0.27$ vs. $1.94, \mathrm{SD} \pm 3.47$; $\mathrm{P}=0.00063$, Mann-Whitney U test) (Fig. 2C). Both the RET mutated and $R E T$ wild-type cases showed significantly lower LRIG1 levels than the normal thyroid glands $(0.41, \mathrm{SD} \pm 0.33$ and $0.34, \mathrm{SD} \pm 0.15$, respectively vs. $1.94, \mathrm{SD} \pm 3.47 ; \mathrm{P}=0.0036$ and $\mathrm{P}=0.0016$, respectively). There was a statistically insignificant association between a higher MTC clinical stage and lower LRIG1 expression ( $\mathrm{P}=0.136$, Kruskal-Wallis test) (Fig. 2D). 

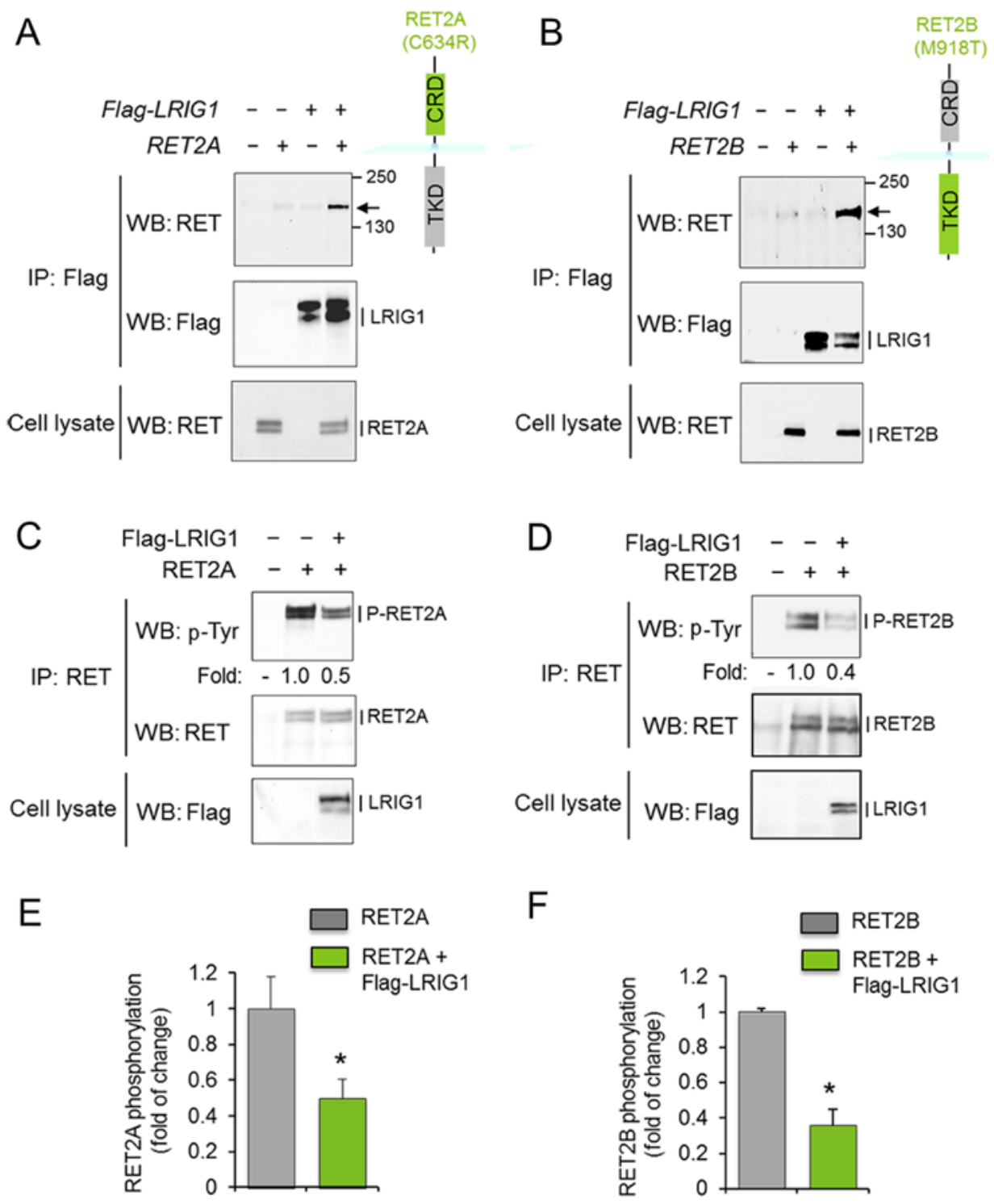

Figure 1. LRIG1 interacts with and restricts the ligand-independent constitutive phosphorylation of RET2A and RET2B oncogenic mutants. A schematic representation of RET2A (i.e., C643R recurrent in MEN2A) and RET2B (i.e., M918T seen in MEN2B and sporadic MTC) are shown at the top. TKD indicates tyrosine-kinase domain and CRD indicates a cysteine-rich domain. (A and B) Co-immunoprecipitation between Flag-tagged LRIG1 (Flag-LRIG1) and RET2A (A) or RET2B (B) overexpressed in COS-7 cells. Cell extracts were analyzed by immunoprecipitation (IP) with anti-Flag antibodies followed by western blotting (WB) with antibodies against RET and reincubation of the same blots with the anti-Flag antibody. The bottom panels show the RET expression in cell lysates. Similar results were obtained in two independent assays. (C and D) Ligand-independent RET2A and RET2B tyrosine phosphorylation in COS-7 cells transfected with either the control vector or Flag-LRIG1. The total lysates were immunoprecipitated with anti-RET antibodies followed by WB with antibodies against phosphotyrosine (p-Tyr). The re-probing of the same blot with anti-RET antibodies is also shown. The bottom panels show the FlagLRIG1 expression in cell lysates. The numbers below the lanes indicate the fold of induction relative to the control and normalized to the total levels of RET protein. (E and F) Bar graphs showing the levels of ligand-independent RET2A (E) and RET2B (F) phosphorylation in the absence or presence of Flag-LRIG1, as assessed in (C and D). The results are shown as the mean \pm SEM of $n=5(E)$ or $n=3(F)$ independent experiments. "P<0.05 by Student's t-test. MTC, medullary thyroid carcinoma; RET, rearranged during transfection; LRIG1, leucine-rich repeats and immunoglobulin-like domains 1.

There was no significant difference in LRIGl levels between the RET-mutated, $R A S$-mutated, or other MTCs.

Cohort3-TMA was analyzed by immunohistochemistry (IHC) using a TMA with 118 thyroid carcinomas (Table I). In this cohort, 125 patients gave informed consent to participate (Table I) and it was possible to perform IHC on samples from 118 patients, whereas for 7 patients, it was not possible to perform this analysis because of the limited amount of available tissue. Examples of cases with different LRIG1 staining intensities and proportions of positive cells are shown in Fig. 3. Detailed data on the clinical parameters and results of IHC for Cohort3-TMA are presented in Table I. The possible associations between LRIG1 immunoreactivity (proportion of positive cells or intensity of staining) and histology, clinical stage, age, sex, and clinical outcome were investigated. A significant difference was found when histology was compared with the percentage of LRIG1-positive cells $\left(\mathrm{P}=0.026, \chi^{2}\right.$ test $)$ and intensity of LRIG1 staining $\left(\mathrm{P}=0.033, \chi^{2}\right.$ test $)$. This showed that the distribution of LRIG1 immunoreactivities (percentage or intensity) was different between the different histological groups. However, because of the small sample sizes, it was not possible to draw firm conclusions about the distributions within individual groups. Both the fraction of LRIG1-positive cells and the intensity of LRIG1 staining were associated with whether 

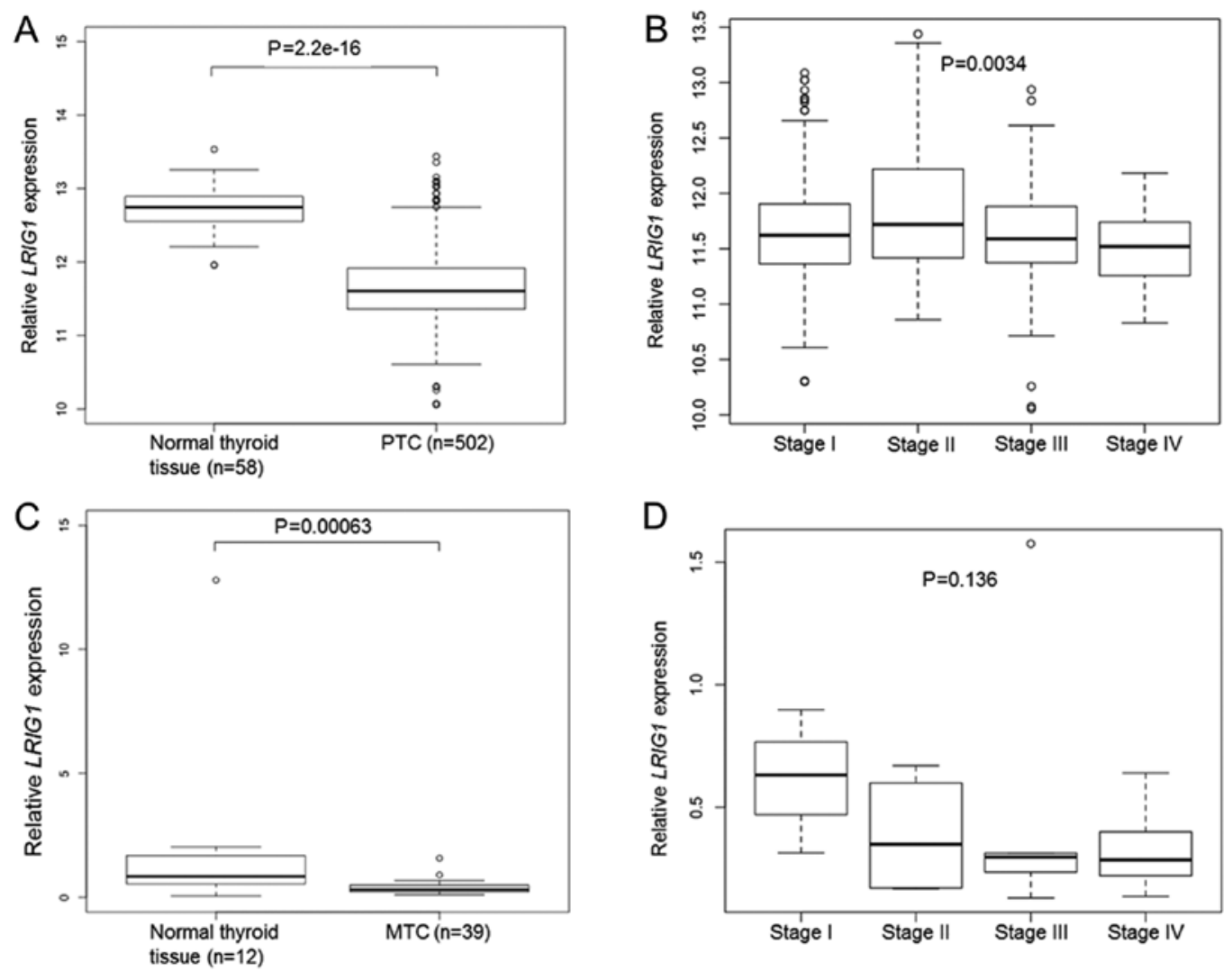

Figure 2. LRIG1 expression levels in PTC, MTC, and normal thyroid tissues. (A) Boxplots showing relative $L R I G 1$ mRNA levels in normal thyroid tissue $(\mathrm{n}=58)$ and PTC $(\mathrm{n}=502)$ according to the TCGA dataset. The $L R I G 1$ expression level was significantly lower in PTC than in normal thyroid tissue $(\mathrm{P}=2.2 \mathrm{e}-16)$. Outlier values are marked ${ }^{\circ}$. (B) Boxplots showing the relative LRIG1 expression in PTC ordered by clinical stage according to the TCGA data set. A higher clinical stage was significantly associated with a lower LRIG1 expression (one-way ANOVA, P=0.0035). (C) Boxplots showing the relative $L R I G 1$ mRNA expression levels in the normal thyroid gland $(\mathrm{n}=12)$ and $\mathrm{MTC}(\mathrm{n}=39)$ measured with quantitative RT-PCR. Shown are the relative $L R I G 1 / R N 18 S$ ratios normalized to the corresponding level in human reference RNA that was arbitrarily set to the value of 1 . The relative $L R I G 1 / R N 18 S$ ratio was significantly lower in MTC than in normal thyroid glands $(\mathrm{P}=0.00063)$. Outlier values are marked ${ }^{\circ}$. (D) Boxplots showing $L R I G 1$ expression in MTC ordered by clinical stage. A higher clinical stage was not significantly associated with a lower $L R I G 1$ expression (Wilcoxon signed-rank test, $\mathrm{P}=0.136$ ). PTC, papillary thyroid carcinoma; MTC, medullary thyroid carcinoma; RET, rearranged during transfection; LRIG1, leucine-rich repeats and immunoglobulin-like domains 1.

the patients were dead or alive at the latest follow-up $(\mathrm{P}=0.036$ and $\mathrm{P}=0.007$, respectively, $\chi^{2}$ test). The patients who died during the follow-up period were older at the time of diagnosis than those still alive $(\mathrm{P}<0.001$, Mann-Whitney $\mathrm{U}$ test $)$, but the age distribution was similar across all categories for the fraction of LRIG1-positive cells and all of the intensities $(\mathrm{P}=0.15$ and $\mathrm{P}=0.12$, respectively, Kruskal-Wallis test). No other significant associations were found between LRIG1 immunoreactivity and the clinical parameters. The same analyses were performed including only PTC and FTC, since these entities have a more favorable clinical course. Both the percentage of positive cells and intensity of staining were still associated with whether the patients were dead or alive at the latest follow-up $(\mathrm{P}=0.014$ and $\mathrm{P}=0.002$, respectively, $\chi^{2}$ test). The median follow-up time was 86 months for PFS, 96 months for DSS, and 96 months for OS. Survival analyses were performed for clinical stage, the percentage of LRIG1-positive cells and the intensity of LRIG1 staining. As expected, the clinical stage was a strong prognostic factor for OS, DSS and PFS, both when comparing all of the stages and when comparing stages I and II with stages III and IV (data not shown). No significant associations were found between LRIG1 immunoreactivity and the cumulative survival rates in Cohort3-TMA, both when analysing the whole cohort as well as when analysing PTC only $(n=72)$. Performing additional sub-group analysis for smaller groups was not relevant due to the limited size of each group.

Animal experiments. To experimentally address the role of LRIG1 in mutated RET-driven MTC, a mouse strain carrying a deficient Lrigl allele was crossed with a transgenic mouse strain that expresses human RET2B in thyroid parafollicular cells (C-cells) and is known to develop MTC or C-cell hyperplasia within 2-years and at high frequencies (13 and $77 \%$, respectively) $(34,35)$. The resulting offspring that were heterozygous for both the Lrigl and the RET $2 B$ alleles were crossed with heterozygous Lrigl mice to yield Lrigl wild-type, heterozygous, or knockout mice with or without the RET2B transgene (Fig. 4A). Only mice that were positive for the RET2B transgene were included in the study. In total, 125 mice were included, of which 46 (37\%) were Lrigl wild-type, 49 (39\%) were Lrigl heterozygous, and 30 (24\%) were Lrig1-deficient. The mice were followed up for up to 2 years. Blood samples were obtained and calcitonin levels were measured every third month beginning at 6 months of age. Surprisingly, the plasma calcitonin levels were below the detection limit of the assay in all of the samples that were analyzed, except for four samples, which were calcitonin positive and represented three different mice. Of the three mice with elevated calcitonin 

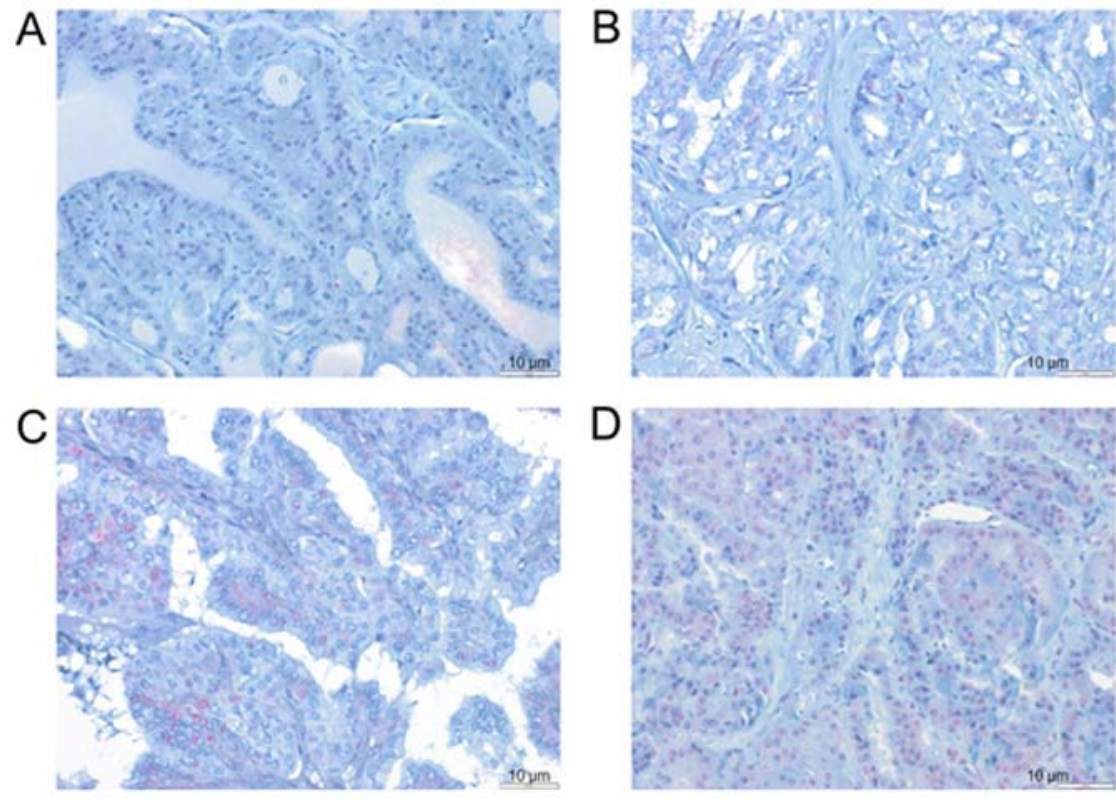

Figure 3. Examples of immunohistochemical stainings of LRIG1 in papillary thyroid carcinomas and their respective scores. (A) A tumor with no positive LRIG1 staining. (B) A tumor with weak LRIG1 staining intensity (1+) and 1-25\% positive cells. (C) A tumor with intermediate LRIG1 staining intensity (2+) and $26-50 \%$ positive cells. (D) A tumor with intermediate LRIG1 staining intensity (2+) and 76-100\% positive cells. Scale bars, $10 \mu \mathrm{m}$. LRIG1, leucine-rich repeats and immunoglobulin-like domains 1 .

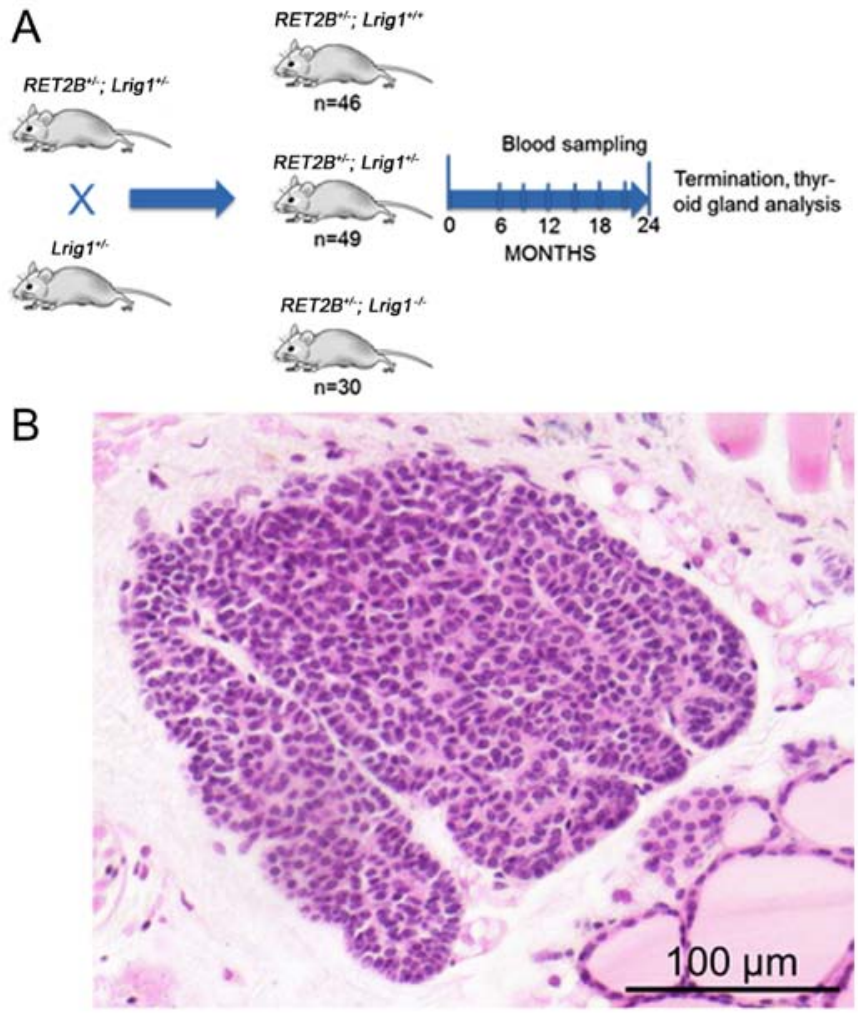

Figure 4. RET2B-induced thyroid gland lesions in mice. (A) A schematic drawing of the mouse experimental set-up. Mice with the genotypes $R E T 2 B^{+/}$; $\operatorname{Lrig}^{+/-}$and $\mathrm{Lrig}^{+/-}$were mated to generate offspring of the genotypes

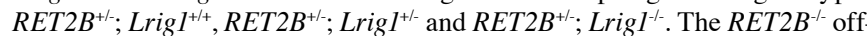
spring were not included in the study. Beginning at 6 months of age, the blood was sampled every $3 \mathrm{rd}$ month and the calcitonin levels were determined. At 24 months of age or at signs of the disease, the mice were sacrificed and their thyroid glands were analyzed. A graphic mouse symbol was downloaded from http://clipartall.com and modified in Photoshop. (B) A micrograph showing an example of a hematoxylin and eosin-stained mouse thyroid gland with apre-invasive lesion. Scale bars, $100 \mu \mathrm{m}$. Lrig1, leucine-rich repeats and immunoglobulin-like domains 1 ; RET, rearranged during transfection. levels, one was Lrigl wild-type and two were heterozygous for the Lrigl allele. Eleven of the 46 wild-type mice (24\%), 8 of the 49 heterozygous mice (16\%), and 7 of the 30 knockout mice $(23 \%)$ died prematurely, that is, before the planned termination at 24 months. For most of the prematurely dead mice, the cause of death was undetermined; therefore, they were excluded from further analysis. At 24 months of age, the remaining mice were sacrificed and their thyroid glands were collected, formaldehyde-fixed, paraffin embedded, sectioned and routinely stained. Pre-invasive lesions were found in 13 of the 35 wild-type glands (37\%), 12 of the 41 heterozygous glands (29\%), and in 11 of the 23 Lrigl-deficient glands (48\%) (Fig. 4B). The remaining 63 thyroid glands showed no evidence of pathological changes. The difference in the incidence of pre-invasive lesions between the wild-type and Lrig1-deficient mice was not statistically significant $(\mathrm{P}=0.42$, $\chi^{2}$ test). Furthermore, no overt cancers were observed.

\section{Discussion}

In this study, the importance of LRIG1 in thyroid cancer was investigated. We showed that LRIG1 physically interacted with and inhibited the activation of the MEN-associated and thyroid cancer-associated mutant RET receptors RET2A and RET2B and was downregulated in human PTC and MTC compared to normal thyroid gland tissues. However, in our mouse model of RET2B-driven MTC, no significant effect of Lrig1 gene ablation was observed.

The interaction between LRIG1 and tyrosine kinase receptors has been described previously for normal RET (27), and here we showed similar interactions between LRIG1 and the mutated and oncogenic RET variants RET2A and RET2B. That is, LRIG1 physically interacted with both RET2A and RET2B, as well as inhibited their constitutive activation as monitored with receptor tyrosine phosphorylation blots. This 
Table I. Clinical features of the patients in Cohort3-TMA.

\begin{tabular}{|c|c|}
\hline Clinical features & No. of cases \\
\hline \multicolumn{2}{|l|}{ TNM stage } \\
\hline I & 53 \\
\hline II & 11 \\
\hline III & 27 \\
\hline IV & 27 \\
\hline \multicolumn{2}{|l|}{ Histology } \\
\hline PTC & 72 \\
\hline FTC & 18 \\
\hline MTC & 5 \\
\hline ATC & 8 \\
\hline PDTC & 4 \\
\hline FTC-oncocytic type & 12 \\
\hline \multicolumn{2}{|l|}{ Deceased } \\
\hline Yes & 65 \\
\hline No & 60 \\
\hline \multicolumn{2}{|c|}{ Deceased due to thyroid cancer } \\
\hline Yes & 25 \\
\hline No & 100 \\
\hline \multicolumn{2}{|l|}{ Relapse } \\
\hline Yes & 37 \\
\hline No & 88 \\
\hline \multicolumn{2}{|c|}{ Intensity of LRIG1 staining } \\
\hline Absent & 28 \\
\hline Weak & 72 \\
\hline Moderate & 18 \\
\hline Intense & 0 \\
\hline \multicolumn{2}{|c|}{ Percentage of LRIG1-positive cells } \\
\hline None & 28 \\
\hline $1-25 \%$ & 18 \\
\hline $26-50 \%$ & 17 \\
\hline $51-75 \%$ & 25 \\
\hline $76-100 \%$ & 30 \\
\hline \multicolumn{2}{|l|}{ Sex } \\
\hline Male & 40 \\
\hline Female & 85 \\
\hline
\end{tabular}

PTC, papillary thyroid carcinoma; FTC, follicular thyroid carcinoma; MTC, medullary thyroid carcinoma; PDTC, poorly differentiated thyroid carcinoma; ACT, anaplastic thyroid carcinoma; LRIG1, leucine-rich repeats and immunoglobulin-like domains 1.

indicates that LRIG1 may play an important role in the initiation or progression of thyroid cancer because mutated RET receptors, including RET2A and RET2B, are established drivers of the majority of MTCs and of some PTCs. However, the RET fusion proteins found in PTC are cytosolic whereas LRIG1 is a transmembrane protein, and whether they functionally interact was not addressed in the present study. Nevertheless, altered signaling pathways in PTC, particularly activation of the MAPK signaling pathway, may be the result of other genetic events, such as activating mutations in BRAF and RAS genes (reviewed in ref. 37). This activation may result in the upregulation of other oncogenic proteins, including other targets of LRIG1, such as the RTKs EGFR and MET. Thus, LRIG1 could be important in PTC through interactions with other proteins as well.

In Cohort1-TCGA, LRIG1 expression data were available for 502 PTC cases but not for other thyroid cancer subtypes; whereas in Cohort2-MTC, we determined the LRIG1 mRNA levels in 39 MTCs. Intriguingly, LRIGl was downregulated in both PTC and MTC compared to normal thyroid gland tissue. Because many PTCs have been reported to have a RET rearrangement and the majority of MTCs de facto showed a mutated $R E T$, the downregulation of LRIG1 expression seen supports the notion that LRIGI downregulation may play a causative role in the etiologies of PTC and MTC. However, as discussed above, this study does not provide evidence for a functional interaction between LRIG1 and the RET fusion proteins found in PTC; however, LRIG1 may possibly exert effects through interactions with other RTKs as well. Another important point is that the use of normal thyroid tissue as control for MTC may not be optimal, but microdissection of parafollicular cells for control was unfortunately not possible to obtain from this material. The IHC analysis of Cohort3-TMA revealed no association between LRIG1 immunoreactivity and patient survival. However, known prognostic factors, such as clinical stage and histological type, were confirmed to be predictive of patient survival in this cohort. Because the majority of cases in Cohort3-TMA (72/119) and all of the cases in Cohort1-TCGA were of the PTC subtype, the data indicate that neither the LRIG1 protein nor the LRIG1 mRNA is prognostic in PTC. Although it could be argued that the material used for the IHC analysis was rather small (118 cases, in total), previous studies of similarly sized cohorts have shown significant associations between LRIG immunoreactivity and patient survival for other types of cancer $(16,17,19,21,38-41)$. Therefore, the importance of the LRIG1 protein and RNA expression levels as prognostic markers in PTC may be of less interest for further study. Because of the relative rarity of MTC, only 5 of the 111 evaluated patients in Cohort3-TMA represented MTC cases, it was not possible to address the role of LRIG1 immunoreactivity as a prognostic marker in MTC in this material. However, since LRIG1 mRNA was downregulated in MTC, it may be of interest to investigate the prognostic value of LRIG1 immunoreactivity in a larger MTC cohort.

The deletion of Lrigl did not affect the apparent tumor incidence or malignancy grade in the mouse model of RET2B-driven MTC. Our hypothesis was that Lrig1, through its suppression of RET2B, would serve as a tumor suppressor in this model system and, therefore, its deletion would enhance the incidence, progression, or severity of the disease. However, no such MTC promoting effects were observed in the Lrigl-deficient mice. One possible explanation for the lack of apparent effects of Lrig1 ablation could be that Lrig1 expression in the wild-type tumors was downregulated through tumor-intrinsic mechanisms, thereby obviating the need for and masking the effects of genetic ablation. This hypothesis is consistent with the strong downregulation of LRIGI expression that was seen in the human MTC series. Regrettably, we were unable to determine whether Lrig1 expression was actually downregulated in the mouse tumors because the Lrigl expression levels could not be determined in the pre-invasive lesions in a reliable manner. Relatively, many of the mice $(24 \%)$ died or had to be sacrificed before the planned 
termination at 24 months of age. In most of these cases, the cause of premature death was undetermined. We do not know if the $R E T 2 B$ transgene caused an increased mortality rate among the experimental mice because no RET $2 B$-negative control colonies were maintained in parallel with the $R E T 2 B$ transgenic experimental group. However, the cause of premature deaths is not likely to have been MTC because the plasma calcitonin levels were analyzed every third month and none of the prematurely dead mice showed increased plasma calcitonin levels prior to their death. In fact, detectable plasma calcitonin levels were observed in only 3 of the $99(3 \%)$ mice. This is in contrast with a previous study, in which up to $90 \%$ of the RET $2 B$-positive mice showed detectable plasma calcitonin levels by the age of 24 months (35). The reason for the observed difference in MTC incidence between the present study and the previous study is not known, but it could possibly be due to differences in the calcitonin assay-sensitivity due to different amounts of plasma available, animal housing conditions, or mouse strain differences. In both studies, the genetic backgrounds of the mice were mixed and thus, presumably not identical. Nevertheless, pre-invasive thyroid gland lesions were found in $37 \%$ of wild-type mice and in $48 \%$ of Lrigl-deficient mice. However, the slightly higher incidence of pre-invasive lesions among the Lrigl-deficient mice was not statistically significant. Thus, no effect of Lrigl-ablation could be demonstrated in our mouse model of RET2B-driven MTC.

In conclusion, our demonstration that LRIG1 negatively regulated the thyroid oncoproteins RET2A and RET2B and was also downregulated in PTC and MTC suggests that LRIG1 plays important roles in thyroid cancer. We believe that the possibility that the downregulation of LRIG1 is a critical tumor-promoting event during papillary and medullary thyroid carcinogenesis and that LRIG1-based therapies could be effective in PTC, MTC and/or other RET-driven cancers warrant further investigations. The concept of possible LRIG1-based therapies has been described in detail in a recent review, highlighting its possible role as a therapeutic inhibitor of receptor tyrosine kinase signaling (42). Further studies based on our findings may initially include cellular and animal studies testing the therapeutic potential of LRIG1 in cellular and animal models of PTC and MTC.

\section{Acknowledgements}

We would like to thank Sjors Fens, Yvonne Jonsson, Annika Holmberg, Charlotte Nordström, and Helena Hermelin for their technical help.

\section{Funding}

This study was supported by grants from the Swedish Society for Medical Research, the Swedish Cancer Society, the Cancer Research Foundation in Northern Sweden, the F Olaison Foundation, and through the regional agreement between Umeå University and Västerbotten County Council in cooperation in the fields of medicine, odontology and health.

\section{Availability of data and materials}

The materials included in the manuscript, including all relevant raw data, will be made freely available to any researchers who wish to use them for non-commercial purposes, while preserving necessary confidentiality and anonymity.

\section{Authors' contributions}

DL, HH and RH coordinated the data collection, were responsible for the animal experiments, the collection of clinical data for cohort 3, carried out the statistical analyses, drafted the manuscript, and provided final approval for the submission. FCA and GP performed the cellular experiments, critically revised the manuscript, and provided final approval for the submission. $\mathrm{CH}$ collected the data for cohort 1 , critically revised the manuscript, and provided final approval for the submission. CL and NW collected the data for cohort 2, critically revised the manuscript, and provided final approval for the submission. JH performed all cacitonin analyses and interpreted the data of these results, critically revised the manuscript, and provided final approval for the submission. MT and TT are senior pathologists and conducted the immunohistochemidal evaluations, critically revised the manuscript, and provided final approval for the submission.

\section{Ethics approval and consent to participate}

The study was approved by the Regional Ethics Review Board in Uppsala (dnr 2010/351). Only patients who provided written informed consent were included in the study.

\section{Consent for publication}

Publication of the clinical datasets in this study does not compromise anonymity or confidentiality or breach local data protection laws.

\section{Competing interests}

The authors declare that they have no competing interests.

\section{References}

1. Ferlay J, Shin HR, Bray F, Forman D, Mathers C and Parkin DM: Estimates of worldwide burden of cancer in 2008: GLOBOCAN 2008. Int J Cancer 127: 2893-2917, 2010.

2. DeLellis R and Williams ED: Tumours of the thyroid and parathyroid. In: World Health Organization Classification of Tumours. Pathology and Genetics of Tumours of Endocrine Organs. DeLellis R and Williams ED (eds). Vol. 8. IARC Press, pp51-56, 2004.

3. Romei C, Fugazzola L, Puxeddu E, Frasca F, Viola D, Muzza M, Moretti S, Nicolosi ML, Giani C, Cirello V, et al: Modifications in the papillary thyroid cancer gene profile over the last 15 years. J Clin Endocrinol Metab 97: E1758-E1765, 2012.

4. Romei C, Ciampi R and Elisei R: A comprehensive overview of the role of the RET proto-oncogene in thyroid carcinoma. Nat Rev Endocrinol 12: 192-202, 2016.

5. Kouvaraki MA, Shapiro SE, Perrier ND, Cote GJ, Gagel RF, Hoff AO, Sherman SI,Lee JE and Evans DB: RET proto-oncogene: A review and update of genotype-phenotype correlations in hereditary medullary thyroid cancer and associated endocrine tumors. Thyroid 15: 531-544, 2005.

6. Nikiforov YE, Rowland JM, Bove KE, Monforte-Munoz H and Fagin JA: Distinct pattern of ret oncogene rearrangements in morphological variants of radiation-induced and sporadic thyroid papillary carcinomas in children. Cancer Res 57: 1690-1694, 1997.

7. Agrawal N AR, Aksoy BA, Ally A, Arachchi H, Asa SL, Auman JT, Balasundaram M, Balu S, Baylin SB, Behera M, et al: Integrated genomic characterization of papillary thyroid carcinoma. Cell 159: 676-690, 2014. 
8. Network CGAR; Cancer Genome Atlas Research Network: Integrated genomic characterization of papillary thyroid carcinoma. Cell 159: 676-690, 2014

9. Guo D, Holmlund C, Henriksson R and Hedman H: The LRIG gene family has three vertebrate paralogs widely expressed in human and mouse tissues and a homolog in Ascidiacea. Genomics 84: 157-165, 2004.

10. Holmlund C, Nilsson J, Guo D, Starefeldt A, Golovleva I, Henriksson R and Hedman H: Characterization and tissuespecific expression of human LRIG2. Gene 332: 35-43, 2004

11. Nilsson J, Vallbo C, Guo D, Golovleva I, Hallberg B, Henriksson R and Hedman H: Cloning, characterization, and expression of human LIG1. Biochem Biophys Res Commun 284: 1155-1161, 2001.

12. Wang Y, Poulin EJ and Coffey RJ: LRIG1 is a triple threat: ERBB negative regulator, intestinal stem cell marker and tumour suppressor. Br J Cancer 108: 1765-1770, 2013.

13. Lindquist D, Kvarnbrink S, Henriksson R and Hedman H: LRIG and cancer prognosis. Acta Oncol 53: 1135-1142, 2014.

14. Krig SR, Frietze S, Simion C, Miller JK, Fry WH, Rafidi H, Kotelawala L, Qi L, Griffith OL, Gray JW, et al: Lrig1 is an estrogen-regulated growth suppressor and correlates with longer relapse-free survival in ER $\alpha$-positive breast cancer. Mol Cancer Res 9: 1406-1417, 2011

15. Willis S, Villalobos VM, Gevaert $\mathrm{O}$, Abramovitz M, Williams C, Sikic BI and Leyland-Jones B: Single Gene Prognostic Biomarkers in Ovarian Cancer: A Meta-Analysis. PLoS One 11: e0149183, 2016.

16. Lindström AK, Ekman K, Stendahl U, Tot T, Henriksson R Hedman H and Hellberg D: LRIG1 and squamous epithelial uterine cervical cancer: Correlation to prognosis, other tumor markers, sex steroid hormones, and smoking. Int J Gynecol Cancer 18: 312-317, 2008.

17. Tanemura A, Nagasawa T, Inui S and Itami S: LRIG-1 provides a novel prognostic predictor in squamous cell carcinoma of the skin: Immunohistochemical analysis for 38 cases. Dermatol Surg 31: 423-430, 2005

18. Lindquist D, Näsman A, Tarján M, Henriksson R, Tot T, Dalianis T and Hedman H: Expression of LRIG1 is associated with good prognosis and human papillomavirus status in oropharyngeal cancer. Br J Cancer 110: 1793-1800, 2014.

19. Sheu JJ, Lee CC, Hua CH, Li CI, Lai MT, Lee SC, Cheng J, Chen CM, Chan C, Chao SC, et al: LRIG1 modulates aggressiveness of head and neck cancers by regulating EGFRMAPK-SPHK1 signaling and extracellular matrix remodeling. Oncogene 33: 1375-1384, 2014.

20. Kvarnbrink S, Karlsson T, Edlund K, Botling J, Lindquist D, Jirström K, Micke P, Henriksson R, Johansson M and Hedman H: LRIG1 is a prognostic biomarker in non-small cell lung cancer. Acta Oncol 54: 1113-1119, 2015.

21. Yang B, Dai C, Tan R, Zhang B, Meng X, Ye J, Wang X, Wei L, $\mathrm{He} \mathrm{F}$ and Chen Z: Lrig1 is a positive prognostic marker in hepatocellular carcinoma. Onco Targets Ther 9: 7071-7079, 2016.

22. Gur G, Rubin C, Katz M, Amit I, Citri A, Nilsson J, Amariglio N, Henriksson R, Rechavi G, Hedman H, et al: LRIG1 restricts growth factor signaling by enhancing receptor ubiquitylation and degradation. EMBO J 23: 3270-3281, 2004.

23. Laederich MB, Funes-Duran M, Yen L, Ingalla E, Wu X, Carraway KL III and Sweeney C: The leucine-rich repeat protein LRIG1 is a negative regulator of ErbB family receptor tyrosine kinases. J Biol Chem 279: 47050-47056, 2004.

24. Miller JK, Shattuck DL, Ingalla EQ, Yen L, Borowsky AD, Young LJ, Cardiff RD, Carraway KL III and Sweeney C: Suppression of the negative regulator LRIG1 contributes to ErbB2 overexpression in breast cancer. Cancer Res 68: 8286-8294, 2008

25. Stutz MA, Shattuck DL, Laederich MB, Carraway KL III and Sweeney C: LRIG1 negatively regulates the oncogenic EGF receptor mutant EGFRvIII. Oncogene 27: 5741-5752, 2008.

26. Shattuck DL, Miller JK, Laederich M, Funes M, Petersen H, Carraway KL III and Sweeney C: LRIG1 is a novel negative regulator of the Met receptor and opposes Met and Her2 synergy. Mol Cell Biol 27: 1934-1946, 2007.
27. Ledda F, Bieraugel O, Fard SS, Vilar M and Paratcha G: Lrig1 is an endogenous inhibitor of Ret receptor tyrosine kinase activation, downstream signaling, and biological responses to GDNF. J Neurosci 28: 39-49, 2008

28. Rondahl V, Holmlund C, Karlsson T, Wang B, Faraz M, Henriksson R and Hedman $\mathrm{H}$ : Lrig2-deficient mice are protected against PDGFB-induced glioma. PLoS One 8: e73635, 2013.

29. Alsina FC, Hita FJ, Fontanet PA, Irala D, Hedman H, Ledda F and Paratcha G: Lrig1 is a cell-intrinsic modulator of hippocampal dendrite complexity and BDNF signaling. EMBO Rep 17: 601-616, 2016

30. Nilsson J, Starefeldt A, Henriksson R and Hedman H: LRIG1 protein in human cells and tissues. Cell Tissue Res 312: 65-71, 2003.

31. Rossel M, Pasini A, Chappuis S, Geneste O, Fournier L, Schuffenecker I, Takahashi M, van Grunsven LA, Urdiales JL, Rudkin BB, et al: Distinct biological properties of two RET isoforms activated by MEN $2 \mathrm{~A}$ and MEN $2 \mathrm{~B}$ mutations. Oncogene 14: 265-275, 1997.

32. Santoro M, Carlomagno F, Romano A, Bottaro DP, Dathan NA, Grieco M, Fusco A, Vecchio G, Matoskova B, Kraus MH, et al: Activation of RET as a dominant transforming gene by germline mutations of MEN2A and MEN2B. Science 267: 381-383, 1995.

33. Wang N, Kjellin H, Sofiadis A, Fotouhi O, Juhlin CC, Bäckdahl M, Zedenius J, Xu D, Lehtiö J and Larsson C: Genetic and epigenetic background and protein expression profiles in relation to telomerase activation in medullary thyroid carcinoma. Oncotarget 7: 21332-21346, 2016.

34. Suzuki Y, Miura H, Tanemura A, Kobayashi K, Kondoh G, Sano S, Ozawa K, Inui S, Nakata A, Takagi T, et al: Targeted disruption of LIG-1 gene results in psoriasiform epidermal hyperplasia. FEBS Lett 521: 67-71, 2002.

35. Acton DS, Velthuyzen D, Lips CJ and Höppener JW: Multiple endocrine neoplasia type 2B mutation in human RET oncogene induces medullary thyroid carcinoma in transgenic mice. Oncogene 19: 3121-3125, 2000.

36. van Veelen W, van Gasteren CJ, Acton DS, Franklin DS, Berger R, Lips CJ and Höppener JW: Synergistic effect of oncogenic RET and loss of p18 on medullary thyroid carcinoma development. Cancer Res 68: 1329-1337, 2008.

37. Xing M: Molecular pathogenesis and mechanisms of thyroid cancer. Nat Rev Cancer 13: 184-199, 2013.

38. Guo D, Nilsson J, Haapasalo H, Raheem O, Bergenheim T, Hedman $\mathrm{H}$ and Henriksson R: Perinuclear leucine-rich repeats and immunoglobulin-like domain proteins (LRIG1-3) as prognostic indicators in astrocytic tumors. Acta Neuropathol 111: 238-246, 2006.

39. Hedman H, Lindström AK, Tot T, Stendahl U, Henriksson R and Hellberg D: LRIG2 in contrast to LRIG1 predicts poor survival in early-stage squamous cell carcinoma of the uterine cervix. Acta Oncol 49: 812-815, 2010.

40. Holmlund C, Haapasalo H, Yi W, Raheem O, Brännström T, Bragge H, Henriksson R and Hedman H: Cytoplasmic LRIG2 expression is associated with poor oligodendroglioma patient survival. Neuropathology 29: 242-247, 2009.

41. Muller S, Lindquist D, Kanter L, Flores-Staino C, Henriksson R, Hedman H and Andersson S: Expression of LRIG1 and LRIG3 correlates with human papillomavirus status and patient survival in cervical adenocarcinoma. Int J Oncol 42: 247-252, 2013.

42. Neirinckx V, Hedman H and Niclou SP: Harnessing LRIG1mediated inhibition of receptor tyrosine kinases for cancer therapy. Biochim Biophys Acta 1868: 109-116, 2017.

This work is licensed under a Creative Common Attribution-NonCommercial-NoDerivatives 4.0 International (CC BY-NC-ND 4.0) License. 